\title{
Plasma $\alpha$-Tocopherol is Negatively Correlated with Hepatocyte Apoptosis in Chronic Hepatitis B Patients
}

\author{
Xiao-Peng Fan ${ }^{1}$, Kai Wang ${ }^{1}$, Yi Liu ${ }^{1}$ and Jie-Fei Wang ${ }^{2}$
}

\begin{abstract}
Aim Hepatocyte apoptosis is involved in the pathogenesis of liver diseases, while at the same time oxidative stress plays an important role in liver cell damage. This prompted us to evaluate the possible relationship between hepatocyte apoptosis and oxidative stress in patients with chronic hepatitis $\mathrm{B}$.

Methods CHB patients were placed in groups A (ALT $>40 \mathrm{IU} / \mathrm{L}$ ) and B (ALT $\leq 40 \mathrm{IU} / \mathrm{L})$. Healthy controls were considered as group C (all ALT $\leq 40 \mathrm{IU} / \mathrm{L}$ ). Serum concentrations of $\alpha$-tocopherol, superoxide dismutase (SOD), glutathione peroxidase (GSH-Px) and malondialdehyde (MDA) were determined and liver cell apoptosis was evaluated by using terminal deoxynucleotydil transferase-mediated d-UTP biotin nick-end labeling (TUNEL).

Results SOD, GSH-Px, and MDA did not differ between groups. $\alpha$-Tocopherol was significantly decreased in groups $\mathrm{A}(\mathrm{p}<0.01)$ and $\mathrm{B}(\mathrm{p}<0.05)$ when compared with group $\mathrm{C}$ and it was negatively correlated with the apoptosis index $(\mathrm{r}=-0.575, \mathrm{p}<0.01)$.

Conclusion Only the plasma concentration of $\alpha$-tocopherol rather than the other oxidative stress markers changed significantly in patients with normal alanine aminotransferase levels (ALT <40 IU/L) when compared with healthy controls and correlated significantly with the apoptosis index, suggesting that $\alpha$ tocopherol may be a possible marker to reflect liver cell damage, especially in the absence of serum aminotransferase elevation.
\end{abstract}

Key words: hepatitis B, chronic, oxidative stress, apoptosis, $\alpha$-tocopherol, TUNEL

(Inter Med 48: 1585-1593, 2009)

(DOI: 10.2169/internalmedicine.48.2336)

\section{Introduction}

Worldwide, there are over 400 million people carrying the hepatitis B surface antigen (HBsAg) (1). As for the long term course and persistent inflammation, patients with chronic hepatitis B (CHB) have a high risk to develop cirrhosis and hepatocellular carcinoma (HCC). Each year more than 1 million people die from these complications (1). However, the exact mechanisms responsible for the pathogenesis in chronic hepatitis B are still unclear. Recently, apoptosis is considered as an important factor influencing the progression of hepatitis (2).

Apoptosis, also termed programmed cell death, has characteristic features and mechanisms which differ from necro- sis (2). Recent studies have demonstrated that hepatocyte apoptosis may not only act as the consequence but also the cause of liver inflammation and fibrosis $(3,4)$. As reported (5), there are up to $30 \%$ of CHB patients having normal serum aminotransferase activity, but most of them often have significant histological evidence of liver damage (6). The present studies found that the progressive liver disease without elevated aminotransferase could be attributed to hepatocyte apoptosis rather than necrotic hepatocyte damage $(2,5)$.

Accumulating evidence has suggested that oxidative stress may play an important role in regulating apoptotic cell death (3). Excessive levels of ROS have been proven to mediate cellular injury and death through mutiple pathways, including lipid peroxidation, alterations in cell signaling, gene

${ }^{1}$ Department of Hepatology, Qilu Hospital of Shandong University, Jinan, China and ${ }^{2}$ Department of ICU, Shanghai Public Health Clinical Center affiliated to Fudan University, Shanghai, China

Received for publication April 5, 2009; Accepted for publication June 2, 2009

Correspondence to Dr. Kai Wang, wangdoc876@yahoo.com.cn, Dr. Jie-Fei Wang, wangjiefei1207@yahoo.com.cn 
expression and DNA damage $(7,8)$. Oxidant-induced apoptosis is not only the simple result of the biochemical interactions of ROS and cellular macromolecules but also is actively regulated by cell signaling cascades (9).

Antioxidants include glutathione (GSH), thioredoxin (TRX), $\alpha$-tocopherol and vitamin C. Reactive oxygen elimination enzymes include superoxide dismutase (SOD), glutathione peroxidase (GSH-Px), and catalase. SOD is induced by oxidative stress and dismutates $\bullet_{2}^{-}$to $\mathrm{H}_{2} \mathrm{O}_{2}$ and oxygen. GSH is a compound belonging to the $\mathrm{SH}$ group and is abundant in the living body. The role of the $\mathrm{SH}$ group is to provide electrons to free radicals to stabilize the radicals. GSH exists in a reduced form in cells. GSH-Px decomposes $\mathrm{H}_{2} \mathrm{O}_{2}$ into water and oxygen with GSH as an electron donor and reduces lipid peroxide to become neutralized.

Oxidative stress has been demonstrated to occur in patients with chronic HBV infection $(10,11)$. Furthermore, numerous studies have proven that oxidative stress induces apoptosis both in vivo and in vitro (12-14). However, the clinical data regarding the relationship between oxidative stress and apoptosis in CHB patients is still limited. Therefore, the present study was aimed to evaluate the relationship between hepatocyte apoptosis and oxidative stress in patients with $\mathrm{CHB}$. In the present study, fifty-eight patients with $\mathrm{CHB}$ and nineteen healthy controls were enrolled. Plasma concentrations of malondialdehyde (MDA), $\alpha$ tocopherol, superoxide dismutase (SOD) and glutathione peroxidase (GSH-Px), being widely validated as oxidative stress parameters, were determined. We also evaluated hepatocyte apoptosis using the terminal deoxynucleotydil transferase-mediated d-UTP biotin nick-end labeling (TUNEL) method.

\section{Patients and Methods}

\section{Patients}

Liver biopsy specimens were obtained from 58 patients with chronic hepatitis B from out-patients of our department and nineteen healthy volunteers from Medical College of Shandong University, from August 2007 to October 2008. The enrolled CHB patients met the diagnosis criteria consisting of the presence of HBsAg and HBV DNA in serum for at least 6 months. The exclusion criteria included the use of interferon or antiviral agent, use of supplemental vitamins, history of diabetes mellitus, coronary artery disease, rheumatoid arthritis, cancer, systemic or local infection, the existence of alcohol intake, poor nutritional status, pregnancy, decompensated and Child B or C cirrhosis, concomitant chronic hepatitis C, hepatitis D, or HIV, or other well known liver diseases such as metabolic or autoimmune disorders and various infectious states of the liver, nonalcoholic steatohepatitis, and HBV-DNA negativity in patients with $\mathrm{CHB}$ and cirrhosis. All clinical studies were performed according to the declaration of Helsinki, and all subjects gave written informed consent before donation.
All of the clinical data of the patients and healthy volunteers is shown in Table 1. There were 57 men and 20 women, mean age being $33 \pm 10$ years (range, 16-51 years). A "normal" ALT level was defined as less than $40 \mathrm{IU} / \mathrm{L}$ at the measurement point, with a persistent state for at least six months. Alanine transaminase (ALT) levels were normal $(\leq$ $40 \mathrm{IU}$ ) in 16 cases and elevated (>40 IU) in 42.

\section{Liver function examination and virological assess- ment}

Routine laboratory methods were used to determine serum biochemical markers of liver disease, including alanine aminotransferase (ALT). Serum HBsAg, hepatitis Be antigen (HBeAg), and anti-HBe were determined quantitatively using electrochemiluminescence immunoassay (ECLIA) on the Roche Elecsys 2010 immunoassay analyzers (Roche, Basel, Switzerland) according to the manufacturer's instructions. Serum viral load of HBV DNA was quantified for each subject with a high sensitivity fluorescent real time quantitatively polymerase chain reaction kit (DaAn Gene Co., Ltd., Guangzhou, P R China) and amplified in a PE5700 fluorescence PCR apparatus (Perkin-Elmer, Norwalk, CT, USA). The results were expressed as HBV DNA copies per milliliter of serum, and the detection sensitivity of the PCR assay was $1 \times 10^{3}$ copies $/ \mathrm{mL}$.

\section{TUNEL assessment of liver cell apoptosis in situ}

Terminal deoxynucleotydil transferase-mediated d-UTP biotin nick-end labeling (TUNEL) was used to detect DNA fragmentation. Proteins were stripped from the nuclei of tissue sections by incubation with proteinase $\mathrm{K}(20 \mu \mathrm{g} / \mathrm{mL}$; Boehringer Mannheim, Mannheim, Germany) for $10 \mathrm{~min}-$ utes at room temperature. Terminal deoxynucleotidyl transferase $(\mathrm{TdT}) \quad(0.3 \quad$ e.u/ $\mu \mathrm{L})$ and digoxigenin-11-UTP (Boehringer Mannheim) in TdT buffer $(30 \mathrm{mmol} / \mathrm{L}$ Trizma base, $\mathrm{pH} 7.2,140 \mathrm{mmol} / \mathrm{L}$ sodium cacodylate, $1 \mathrm{mmol} / \mathrm{L}$ cobalt chloride) were added to the sections before incubation in a humid atmosphere at $37^{\circ} \mathrm{C}$ for 60 minutes. Before diaminobenzidine staining, the sections were incubated with peroxidase-conjugated Fab fragments of anti-digoxigenin antibody (Boehringer Mannheim) in phosphate buffer (1: 50). All solutions were prepared with DNAse-free distilled water and molecular biology grade reagents to avoid false positive results due to DNAse contamination. Sections pretreated for 10 minutes with $0.7 \mathrm{mg} / \mathrm{mL}$ DNAse I (Boehringer Mannheim) in sodium cacodylate buffer ( $\mathrm{pH}$ 7.2) served as positive controls. After thorough washing in distilled water, the slides were processed according to the above protocol. TdT was omitted for the negative controls. TUNEL evaluation was performed without knowledge of the patient's clinical and histopathologic data. At least 5,000 hepatocytes in each specimen were evaluated for DNA fragmentation. The proportion of labeled cells counted, using a microscope with an eyepiece grid, was expressed as a percentage of the total number of cells and was defined as the apoptotic index (AI). Stained hepatocytes at the edge of biopsy specimens 
Table 1. Characteristics of the Study Groups

\begin{tabular}{|c|c|c|c|}
\hline Characteristic & $\begin{array}{l}\text { Patient } \\
(\mathrm{n}=58)\end{array} \quad$ group & $\begin{array}{l}\text { Control group } \\
(\mathrm{n}=19)\end{array}$ & $\mathrm{p}$ value \\
\hline Sex (male/female) & $45 / 13$ & $12 / 7$ & \\
\hline Age (years) & & & \\
\hline Mean \pm SEM & $33 \pm 1.34$ & $30 \pm 1.83$ & \\
\hline $\operatorname{ALT}(\mathrm{IU} / \mathrm{L})$ & & & \\
\hline$>40 \mathrm{IU} / \mathrm{L}$ (elevated) & $\mathrm{n}=42$ & & \\
\hline$\leqslant 40 \mathrm{IU} / \mathrm{L}($ normal $)$ & $\mathrm{n}=16$ & $\mathrm{n}=19$ & \\
\hline Mean \pm SEM & $105.34 \pm 13.92$ & $24.37 \pm 2.09$ & 0.000 \\
\hline HBV DNA (copies/mL) & & & \\
\hline$\geqslant 105($ copies $/ \mathrm{mL})$ & $\mathrm{n}=32$ & & \\
\hline$\leqslant 105($ copies $/ \mathrm{mL})$ & $\mathrm{n}=36$ & $\mathrm{n}=19$ & \\
\hline $\mathrm{HBeAg}$ & & & \\
\hline Positive & $\mathrm{n}=21$ & & \\
\hline Negative & $\mathrm{n}=37$ & $\mathrm{n}=19$ & \\
\hline Inflammation grade & & & \\
\hline G0 & $\mathrm{n}=4(6.9 \%)$ & & \\
\hline G1 & $\mathrm{n}=37(63.8 \%)$ & & \\
\hline $\mathrm{G} 2$ & $\mathrm{n}=13(22.4 \%)$ & & \\
\hline G3 & $\mathrm{n}=4(6.9 \%)$ & & \\
\hline G4 & $\mathrm{n}=0(0 \%)$ & & \\
\hline Fibrosis stage & & & \\
\hline 0 & $\mathrm{n}=12(20.7 \%)$ & & \\
\hline 1 & $\mathrm{n}=22(37.9 \%)$ & & \\
\hline 2 & $\mathrm{n}=18(31.0 \%)$ & & \\
\hline 3 & $\mathrm{n}=4(6.9 \%)$ & & \\
\hline 4 & $\mathrm{n}=2(3.4 \%)$ & & \\
\hline $\begin{array}{l}\text { Apoptosis index(apoptotic cells } \\
\text { number/total cells number } \times 400 \text { ) }\end{array}$ & & & \\
\hline Mean \pm SEM & $7.60 \pm 0.43$ & 0 & 0.000 \\
\hline Plasma $\alpha$-tocopherol levels $(\mu \mathrm{g} / \mathrm{mL})$ & & & \\
\hline $\begin{array}{c}\text { Mean } \pm \text { SEM } \\
\text { plasma GSH-Px levels(AU) }\end{array}$ & $6.72 \pm 0.29$ & $9.95 \pm 0.29$ & $<0.005$ \\
\hline $\begin{array}{l}\text { Mean } \pm \text { SEM } \\
\text { plasma concentration of SOD }(\mathrm{U} / \mathrm{mL})\end{array}$ & $66.34 \pm 1.45$ & $75.38 \pm 1.83$ & $<0.005$ \\
\hline $\begin{array}{l}\text { Mean } \pm \text { SEM } \\
\text { plasma levels of MDA }(\mathrm{nmol} / \mathrm{mL})\end{array}$ & $78.76 \pm 2.09$ & $91.92 \pm 2.52$ & $<0.005$ \\
\hline Mean \pm SEM & $6.10 \pm 0.16$ & $4.91 \pm 0.27$ & $<0.005$ \\
\hline
\end{tabular}

Data were presented as Mean \pm SEM. $\mathrm{p}$ values were performed by t-test between the two groups. ALT, alanine aminotransferase; GSH-Px, glutathione peroxidase; MDA, malondialdehyde; SOD, superoxide dismutase; GSH-Px, glutathione peroxidase;

SEM, standard error of the mean

were not counted and the number of hepatocytes present in a 7-mm biopsy specimen was chosen as a maximum common reference number. Apoptotic bodies were not considered in the apoptotic index calculation as this would result in an overestimation of the number of apoptotic cells.

\section{Determination of oxidative stress parameters}

Blood samples were collected from all 77 subjects and $\alpha-$ tocopherol was measured in heparinized plasma. Samples were protected from light during processing and stored at $70^{\circ} \mathrm{C}$ prior to analyses. Assays were performed using a micromethod on $50 \mathrm{Ml}$ aliquots with reversed-phase HPLC and detection by fluorescence at 292/324 nm. The precision between series was $5.0 \mathrm{CV} \%$. $\alpha$-Tocopherol concentration was expressed as $\mu \mathrm{g} / \mathrm{mL}$.

Plasma malondialdehyde (MDA) was estimated colorimetrically by a thiobarbituric acid assay procedure (15). In brief, $0.1 \mathrm{~mL}$ of plasma/liver homogenate was treated with 2 $\mathrm{mL}$ of $(1: 1: 1$ ratio) TBA-TCA-HCl reagent (TBA, $0.37 \%, 0.25 \mathrm{~N} \mathrm{HCl}$ and $15 \% \mathrm{TCA}$ ) and placed in a water bath for 15 minutes, cooled and centrifuged and then clear supernatant was measured at $535 \mathrm{~nm}$ against blank. MDA was calibrated using 1,1,3,3-tetraethoxypropane (Sigma Chemical, St. Louis, MO) as a standard. Results were expressed as $\mathrm{nmol} / \mathrm{mL}$.

SOD activity was detected by the nitroblue tetrazolium (NBT) reduction by $\mathrm{O}_{2}^{-}$generated by the xanthine/xanthine oxidase system (16). SOD activity was measured at $560 \mathrm{~nm}$ by detecting the inhibition of this reaction. One SOD unit was defined as the enzyme amount causing 50\% inhibition in the $\mathrm{NBTH}_{2}$ reduction rate. SOD activity was also expressed as $\mu / \mathrm{mL}$.

GSHPx activity in plasma was measured according to the method of Paglia and Valentine (17). Enzyme activity was determined from the oxidation of reduced NADPH in the presence of $\mathrm{H}_{2} \mathrm{O}_{2}$ used as substrate. The decrease in concentration of NADPH was monitored and recorded at $340 \mathrm{~nm}$ in a mixture containing reduced glutathione and glutathione reductase. Enzyme units were defined as the number of micromoles of NADPH oxidized per minute. Results were de- 


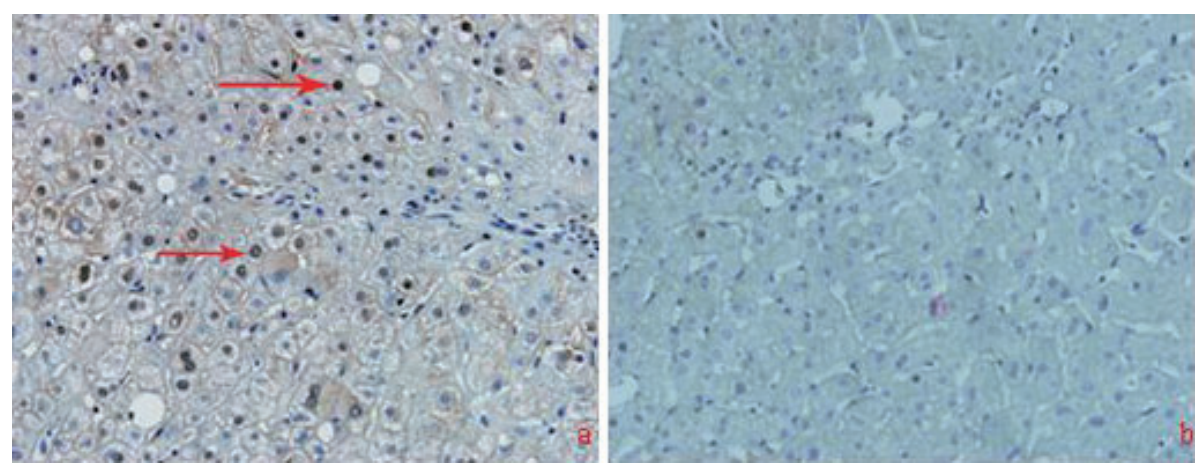

Figure 1. Apoptosis in the liver of controls and patients with chronic hepatitis B. DNA fragmentation was assessed on deparaffinized liver tissue section by terminal deoxynucleotydil transferasemediated d-UTP biotin nick-end labeling (TUNEL). The fragmented DNA were stained dark brown (arrow). Original magnification $\times \mathbf{4 0 0}$.

fined as $\mathrm{AU}$.

\section{Histopathology assessment}

All liver biopsy specimens were fixed in Bouin's solution and embedded in paraffin for routine staining with hematoxylin-eosin-saffron, followed by the evaluation of two experienced pathologists using the Scheuer score who were blinded to the clinical and laboratory observation. The liver histology of the patients was graded and staged by the modified KNODELL scoring system (18).

\section{Statistical analysis}

Continuous normally distributed variables were represented graphically as mean \pm standard error (SEM). To compare the means between groups, one-way analysis of variance (ANOVA) or t-test was performed. The post hoc comparisons were performed using Duncan's multiple test. To determine differences between groups not normally distributed, medians were compared using Kruskal-Wallis ANOVA or the Mann-Whitney U test. The degree of association between variables was assessed using Spearman's nonparametric correlation. All analyses were carried out by using SPSS software version 13.0 (SPSS Inc. Chicago, IL, USA). A $p$-value of less than 0.05 was considered to be statistically significant.

\section{Results}

\section{In situ detection of apoptosis in liver biopsies}

Apoptotic cells were identified by TUNEL in conjunction with characteristic morphological changes, such as cell shrinkage, membrane blebbing, and chromatin condensation, to distinguish apoptotic cells and apoptotic bodies from necrotic cells. We observed TUNEL-positive hepatocytes in all 58 biopsy specimens from patients with chronic hepatitis B, but none or rare in 20 controlled liver specimens. Apoptotic bodies were only detected within the Kupffer cells, with strong staining of shrunken nuclear chromatin fragments, and were not calculated because it might overrate the degree of apoptosis. Apoptotic cells and bodies were seen both in the periportal and intralobular areas (Fig. 1), but tended to cluster at the vicinity of infiltrating lymphocytes. We used the apoptosis index to assess the TUNEL-positive cells in liver specimens which was expressed as the ratio of apoptotic hepatocytes to the total cells per high power $(\times 400)$ in at least five random microscopic fields for each specimen. The apoptotic index of hepatic cells in patients with chronic hepatitis $B$ ranged from 1.77 to 14.86 (mean \pm SEM;7.60 \pm 0.43 ), which is significantly higher than normal controls.

CHB patients were placed into two groups: group A with elevated ALT activity (ALT $>40$ IU/L), group B with normal ALT activity (ALT $\leq 40$ IU/L). Healthy controls were considered as group C. The AI values of subjects in group B were significantly lower than group A $(\mathrm{p}=0.045)$. No association was obtained between AI values and serum ALT activity ( $\mathrm{p}=0.828$ ).

\section{Relation of apoptosis with histopathologic, bio- chemical and virological parameters in patients with chronic HBV infection}

The mean number of TUNEL-positive cells in different grades of inflammation is shown in Table 1. In control liver cells TUNEL-positive cells were rarely labeled. Statistical analysis of 58 patients with different grades of disease activity revealed that AI correlated positively with the grade of inflammation $(\mathrm{p}<0.001)$ (Fig. 2a).

We did not find any significant difference between AI values of patients with variable stages of fibrosis $(\mathrm{p}=0.091)$ (Fig. 2b). Furthermore, no significant correlation between AI and serum transaminase levels $(\mathrm{p}=0.828)$, the viral load or HBV genotype (data not shown) was found.

There was no significant difference of AI values between HBeAg-positive patients and HBeAg-negative patients too $(p>0.05)$. Thus, the positive correlation of AI with the necroinflammatory activity suggests that apoptosis might be involved in the $\mathrm{HBV}$-associated immune response and liver injury. 

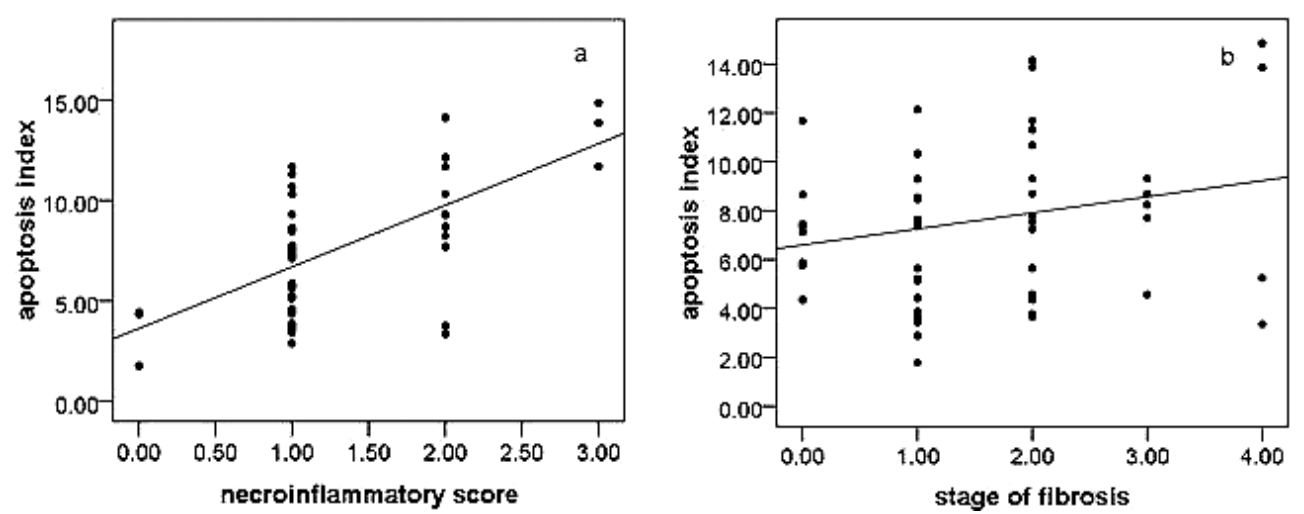

Figure 2. (a) Significant negative correlation between apoptosis indices and necroinflammation grades $(\mathbf{p}<0.01)$ and (b) no significant correlation between apoptosis indices and fibrosis stages $(p>0.05)$. The regression lines are plotted.
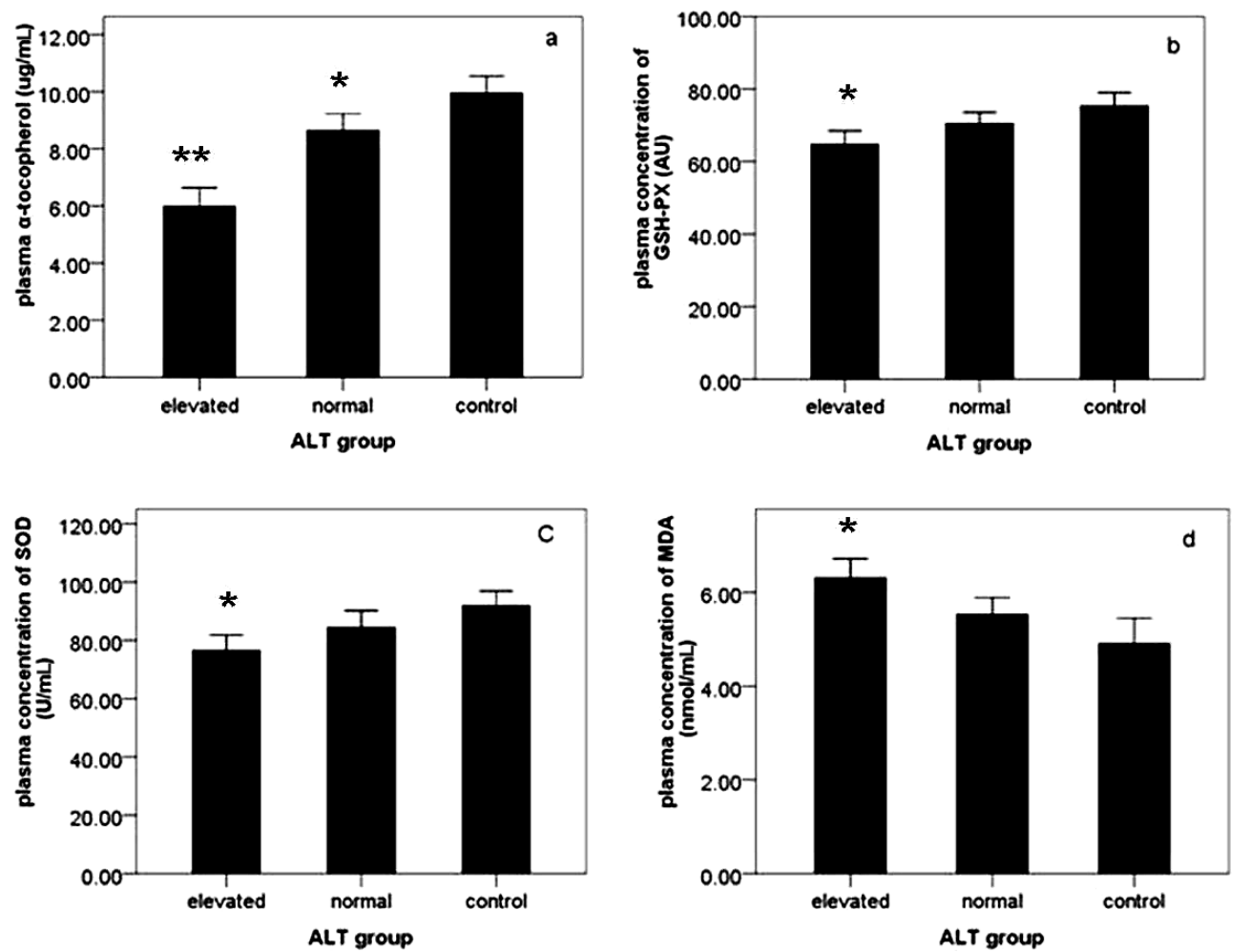

Figure 3. $\alpha$-Tocopherol, SOD, GSH-Px and MDA levels in plasma of individuals of groups A (elevated), B (normal), C (control). All of the marker values were obviously decreased in group A when compared with group $\mathbf{C}$, but only $\alpha$-tocopherol changed significantly in group $\mathbf{B}$ when compared with group $C$. Values are expressed as means \pm standard error of the mean. $* p<0.05$, $* * \mathbf{p}<0.01$ as compared with group $\mathbf{C}$ (one-way analysis of variance/Duncan).

\section{Relation between oxidative stress indicators and histopathologic, biochemical and virological pa- rameters in patients with chronic $\mathrm{HBV}$ infection}

We measured the levels of $\alpha$-tocopherol, GSH-Px, SOD and MDA in plasma from 58 patients with chronic HBV infection and 19 healthy controls. In CHB patients, plasma levels of $\alpha$-tocopherol, GSH-Px, SOD decreased while MDA increased significantly when compared with control subjects (all $p$ values $<0.005$ ). Significant correlations were seen between all these markers and necroinflammatory grades and fibrosis stages (all $p$ values $<0.01$ ). But, we did not find an association of these indicators with the viral load or HBV genotype but observed a negative correlation with ALT activity (all $p$ values $<0.05$ ) in CHB patients. One-way analysis of variance (ANOVA) revealed that all these oxidative stress markers differed significantly among groups A, B and $\mathrm{C}$ according to ALT activity $(\mathrm{p}<0.05)$ as shown by post hoc comparisons, all these parameters obviously differed in group A when compared with group $\mathrm{C}$, but when compared with $\mathrm{B}$, only $\alpha$-tocopherol values increased significantly in group C (Fig. 3) $(\mathrm{p}<0.05)$. 

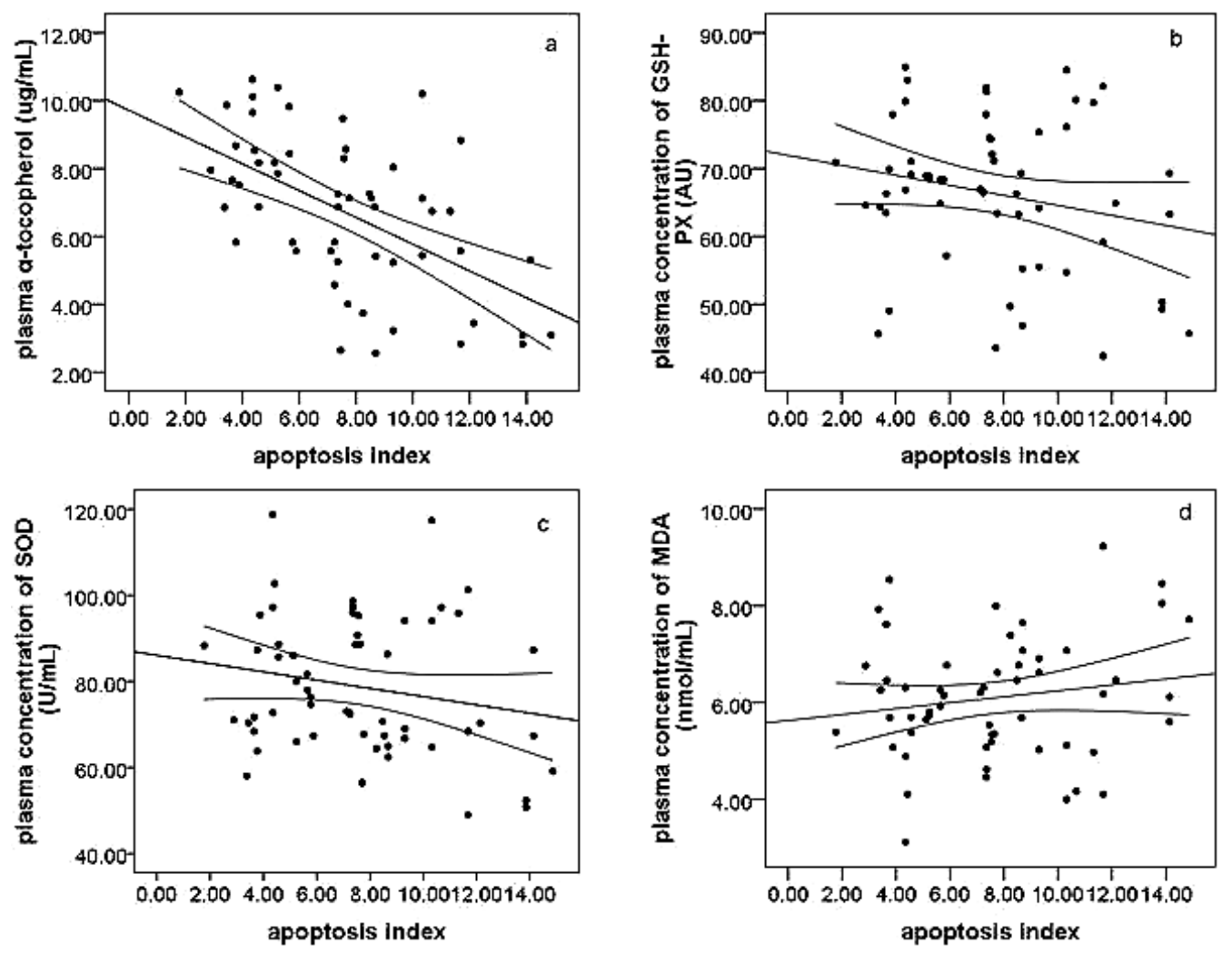

Figure 4. (a) Significant positive correlation between apoptosis index and $\alpha$-tocopherol levels in plasma is shown $(\mathbf{p}<\mathbf{0 . 0 1})$. (c, $\mathrm{d}$ e) No significant correlation between apoptosis index and the concentration of GSH-Px, SOD or MDA in plasma was obtained (all $\mathrm{p}>\mathbf{0 . 0 5}$ ). The regression line and the $95 \%$ confidence interval are plotted.

\section{Correlation of plasma oxidative stress indicators with hepatocyte apoptosis in patients with chronic HBV infection}

Pearson analysis was used to study the relationship between the $\alpha$-tocopherol, GSH-Px, SOD and MDA and hepatocyte apoptosis. As shown in Fig. 4, a positive correlation was obtained between hepatic apoptosis index and the concentration of $\alpha$-tocopherol $(\mathrm{r}=-0.575 \mathrm{p}<0.01)$ but there was no correlation with other indicators. Furthermore, in this section, we also classified the CHB patients into three groups according to their AI values. There were 15 patients with $\mathrm{AI} \leq 5$ in group 1,29 5patients with $5<\mathrm{AI} \leq 10$ in group 2 and 14 patients with AI $>10$ in group 3. One-way ANOVA and post hoc test revealed that only the concentration of $\alpha$-tocopherol was significantly different among the study groups. As shown in Fig. 5, the values of $\alpha$ tocopherol level were decreased significantly in groups 2 and 3 when compared with group 1.

\section{Discussion}

In our study, we found that most hepatitis B patients have evidence of inflammation or fibrosis with a normal alanine aminotransferase level $(6,19)$. Thus "normal" aminotransferase activity may not be considered the same as "healthy" aminotransferase activity (20). Actually, during liver dam- age, ALT is released into the peripheral blood mainly from necrotic hepatocytes, whereas apoptotic liver cells are thought not to release the cell contents because of the integrity of the envelope enclosing the fragments (21). Thus, the undetectable ALT elevation, could not exclude larvaceous liver injury. According to previous reports $(22,23)$, we observed that hepatocyte apoptosis indices in CHB patients were conspicuously higher than in healthy controls, and patients without elevated aminotransferase activity also had notable apoptotic hepatocytes when compared with controls. No significant association of hepatocyte apoptosis with serum aminotransferase activity was obtained. It suggested that chronic hepatocyte damage could occur in HBVinfected patients without overt biochemical changes. Then the latent progressive liver injury, such as in asymptomatic carriers, might at least be partly attributed to hepatocyte apoptosis.

However, in some circumstances, apoptotic process could excite a secondary inflammation and necrosis and lead to the release of transaminases in serum after an apoptotic stimulus (24).

A strong correlation with the liver inflammation grades was obtained and apoptotic cells and bodies were observed tending to cluster at the vicinity of infiltrating lymphocytes supporting the hypothesis of an immune-mediated mechanism of apoptotic hepatocyte damage in chronic hepatitis B $(25,26)$. Recent studies proposed that apoptosis plays a 

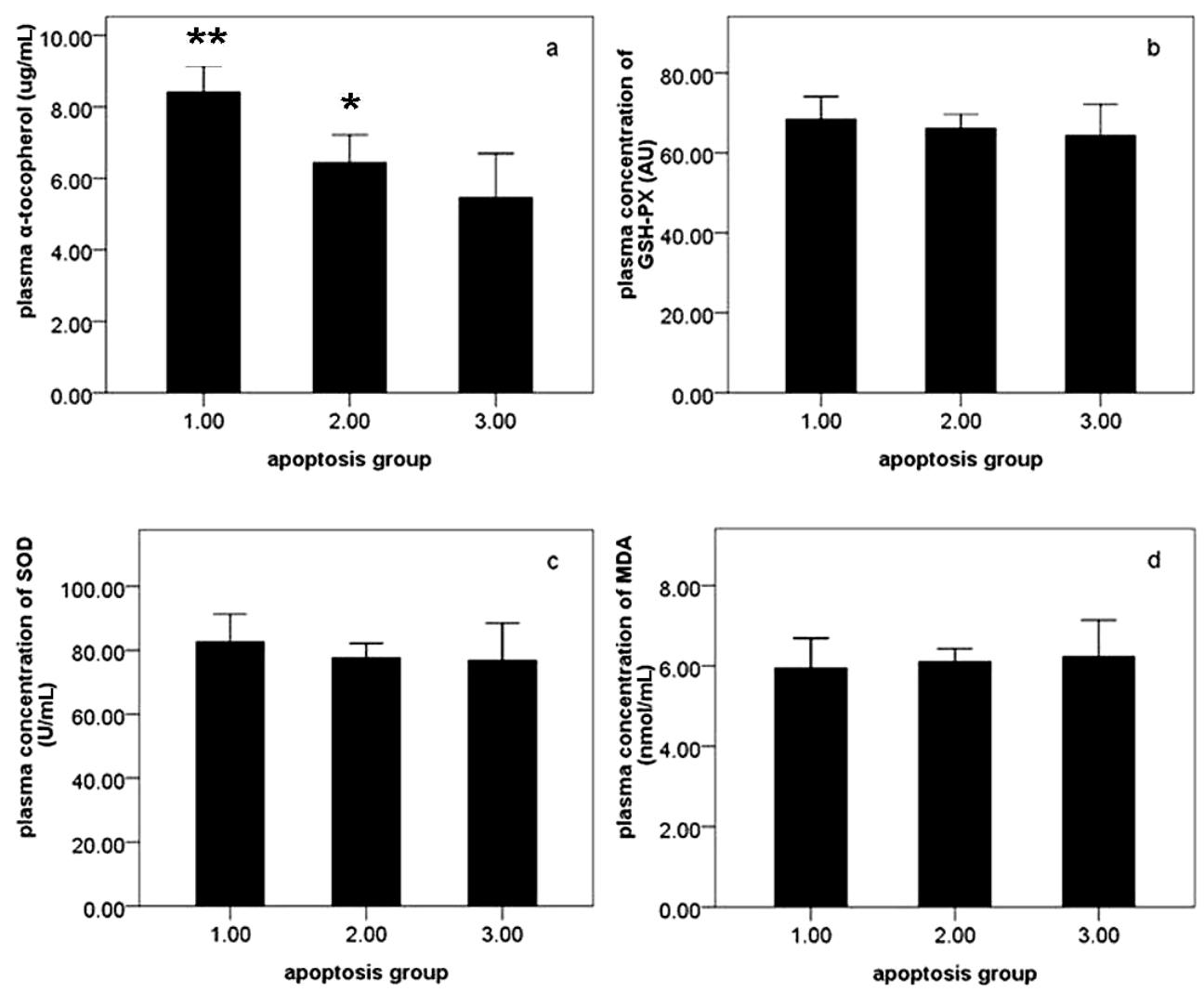

Figure 5. SOD, MDA, GSH-Px and $\alpha$-tocopherol levels in plasma of patients in different apoptosis groups. Values are expressed as means \pm standard error of the mean. Only the values of the $\alpha$ tocopherol level were significantly different among the study groups. ${ }^{*} \mathbf{p}<0.05, * * \mathbf{p}<0.01$ as compared with group 3 (one-way analysis of variance/Duncan).

role and is not only a consequence in the liver inflammation (27). Excessive apoptosis exceeding the capacity of the liver to clear debris and apoptotic bodies may cause the release of contents, induce tissue damage and elicit an inflammatory response (28).

There are some findings that apoptotic hepatocytes could also trigger liver fibrosis $(10,29)$. A new observation demonstrated that phagocytosis of hepatocyte apoptotic bodies by stellate cells could induce procollagen- $\alpha 1$ (I) and TGF- $\beta$ expression in vitro, and the mechanism may be involved in the NADPH oxidase activation and superoxide production (10). However, in the present study, we did not find any association between the apotosis index and the stage of fibrosis. It could be attributable to the long-term course of hepatitis and other multi-factors influencing fibrosis such as viral load and viral genotypes.

Actually, the obtained results showed that no significant correlation was found between AI and HBV DNA, and ALT level. Moreover, we did not find the correlation of AT and HBe status, suggesting that the occurrence of intrahepatic apoptosis might be involved in multiple complicated mechanisms, not only viral factor itself. In other words, HBV itself could not cause the appearance of intrahepatic apoptosis in patients with chronic hepatitis $\mathrm{B}$, at least in part, although HBx protein has been demonstrated to be capable of inducing apoptosis in many experimental conditions $(30,31)$.

Oxidative stress is reported to trigger liver cell apopto- sis $(12,14,32)$ and aggravate the inflammation and fibrosis of hepatitis which can be prevented by antioxidants $(33,34)$. In the present study, we investigated the concentration of several plasma oxidative stress markers including $\alpha$ tocopherol (one of the 8 isomers of vitamin E), MDA, SOD and GSH-Px (two enzyme-antioxidants in cells). Significant differences of $\alpha$-tocopherol, MDA, GSH-Px and SOD in CHB patients were observed when compared with controls and an obvious association between serum aminotransferase activity and these markers values was found. These results may indicate that the progressive hepatitis and liver cell damage were related to decreased levels of endogenous antioxidants. $\alpha$-Tocopherol is a lipid soluble vitamin and it is well known as a free-radical-scavenging antioxidant (35). Of interest, compared with group $\mathrm{C}$, we observed that serum levels of $\alpha$-tocopherol were significantly decreased in group $\mathrm{B}$, but the other antioxidants (SOD, GSH-Px) were not altered significantly. Furthermore, we did not find a significant alteration in the lipid peroxidation marker (MDA) between groups B and C either. Our results indicated that $\alpha$ tocopherol might act as an earlier-active antioxidant prior to other enzymatic antioxidants and that lipid peroxidation started only after $\alpha$-tocopherol had been consumed. Previous studies have demonstrated that $\alpha$-tocopherol inhibits lipid peroxidation and increases other enzyme-antioxidant activities in different experimental models of liver injury (36). von Herbay et al and Rocchi et al $(37,38)$ also observed a 
negative correlation between vitamin $\mathrm{E}$ and disease severity in patients with viral hepatitis.

In the present study, we found $\alpha$-tocopherol, except for MDA, SOD and GSH-Px was significantly decreased among the three apoptosis groups. Moreover a significant correlation was obtained between hepatocyte apoptosis index and the concentration of plasma $\alpha$-tocopherol but not other markers. There have been studies demonstrating that the apoptotic process was triggered by moderate oxidative stimuli, whereas overwhelming oxidative stress may lead to more severe cell damage such as necrosis $(39,40)$. Thus, our findings may illustrate that $\alpha$-tocopherol is less resistant to the early stage of oxidative stress than other enzyme antioxidants. However, the anti-apoptotic effect of $\alpha$-tocopherol was thought to be not only due to its antioxidative property which directly inhibits free radical-mediated apoptosis. Allen and Tresini (41) declared that apart from the antioxidative property, $\alpha$-tocopherol could regulate the expression of several genes and signals responsible for cell death. Previous studies declared that the cytoprotective effects of $\alpha$ tocopherol might not be related to the antioxidant action, but rather to the effects of the decreased the NF- $\kappa \mathrm{B}$ dependent NOS-2 expression and enhanced PPAR- $\alpha$-associated CPT1 expression (42). This may be another possible explanation for our results. Hepatic fibrosis stages associated well with decreased antioxidants and increased MDA levels, suggesting the contribution of ROS to the progression of liver fibrosis.
There are still some limitations in our present study. First, as a matter of fact, patients with the "normal" ALT level are defined as less than $40 \mathrm{IU} / \mathrm{L}$ at the measurement point, with the persistent state at least for six months. However, the state of ALT of less than $40 \mathrm{IU} / \mathrm{L}$ for at six months, is not suitable to represent a "normal" condition, according to the report of Tanaka et al (43). Second, it is unclear whether or not the finding is specific for chronic hepatitis B. For example, non-alcoholic steatohepatitis (NASH) is thought to be mediated by oxidative stress $(44,45)$. In future research, it will be of great interest to initiate such work to identify the specificity for chronic hepatitis B.

In conclusion, we suggest that apoptosis might not only be a consequence but it may also be the cause of the progression of liver inflammation in patients with chronic hepatitis B. $\alpha$-Tocopherol may be a potential biomarker that could predicate apoptotic hepatocyte damage and indicate disease severity especially in the absence of serum aminotransferase alteration; however, in order to draw confirm this hypothesis, much more investigation is necessary. The application of $\alpha$-tocopherol might attentuate liver cell damage and thus diminish the progression of hepatic inflammation and fibrosis.

\section{Acknowledgement}

The present study was supported by grants from the provincial scientific and technical key project of Shandong Province (2006GG2202042).

\section{References}

1. Sugiyama M, Tanaka Y, Kato T, et al. Influence of hepatitis B virus genotypes on the intra- and extracellular expression of viral DNA and antigens. Hepatology 44: 915-924, 2006.

2. Bantel H, Lugering A, Poremba C, et al. Caspase activation correlates with the degree of inflammatory liver injury in chronic hepatitis C virus infection. Hepatology 34: 758-767, 2001.

3. Czaja MJ. Induction and regulation of hepatocyte apoptosis by oxidative stress. Antioxid Redox Signal 4: 759-767, 2002.

4. Foster GR. Apoptotic cell death: the caspase-cleavage "gold rush". Lancet 365: 1293-1294, 2005.

5. Volkmann X, Fischer U, Bahr MJ, et al. Increased hepatotoxicity of tumor necrosis factor-related apoptosis-inducing ligand in diseased human liver. Hepatology 46: 1498-1508, 2007.

6. Bantel H, Lugering A, Heidemann J, et al. Detection of apoptotic caspase activation in sera from patients with chronic HCV infection is associated with fibrotic liver injury. Hepatology 40: 10781087, 2004.

7. Haddad JJ. Redox and oxidant-mediated regulation of apoptosis signaling pathways: immuno-pharmaco-redox conception of oxidative siege versus cell death commitment. Int Immunopharmacol 4: 475-493, 2004.

8. Jaeschke H. Reactive oxygen and mechanisms of inflammatory liver injury. J Gastroenterol Hepatol 15: 718-724, 2000.

9. Singh R, Czaja MJ. Regulation of hepatocyte apoptosis by oxidative stress. J Gastroenterol Hepatol 22 Suppl 1 : S45-S48, 2007.

10. Zhan SS, Jiang JX, Wu J, et al. Phagocytosis of apoptotic bodies by hepatic stellate cells induces NADPH oxidase and is associated with liver fibrosis in vivo. Hepatology 43: 435-443, 2006.

11. Bonkovsky HL, Banner BF, Rothman AL. Iron and chronic viral hepatitis. Hepatology 25: 759-768, 1997.

12. Matsumaru K, Ji C, Kaplowitz N. Mechanisms for sensitization to TNF-induced apoptosis by acute glutathione depletion in murine hepatocytes. Hepatology 37: 1425-1434, 2003.

13. Minana JB, Gomez-Cambronero L, Lloret A, et al. Mitochondrial oxidative stress and CD95 ligand: a dual mechanism for hepatocyte apoptosis in chronic alcoholism. Hepatology 35: 1205-1214, 2002.

14. Kurose I, Higuchi H, Miura S, et al. Oxidative stress-mediated apoptosis of hepatocytes exposed to acute ethanol intoxication. Hepatology 25: 368-378, 1997.

15. Albro PW, Corbett JT, Schroeder JL. Application of the thiobarbiturate assay to the measurement of lipid peroxidation products in microsomes. J Biochem Biophys Methods 13: 185-194, 1986.

16. Shinohara R, Ogishi K, Nagamura N, et al. [Application of superoxide dismutase to clinical chemistry. 1. Simple colorimetric determination method of human serum superoxide dismutase (author's transl)]. Rinsho Byori 24: 926-930, 1976.

17. Paglia DE, Valentine WN, Nakatani M, et al. AMP deaminase as a cell-age marker in transient erythroblastopenia of childhood and its role in the adenylate economy of erythrocytes. Blood 74: 2161$2165,1989$.

18. Okuno $T$, Shindo $M$, Arai K, et al. Histological improvement of chronic hepatitis $\mathrm{B}$, and non-A, non-B with interferon treatment: application of a numerical scoring system for evaluating sequential morphologic changes. Gastroenterol Jpn 25: 70-77, 1990.

19. Karmen A, Wroblewski F, Ladue JS. Transaminase activity in human blood. J Clin Invest 34: 126-131, 1955.

20. Zeuzem S, Diago M, Gane E, et al. Peginterferon alfa-2a (40 kilo- 
daltons) and ribavirin in patients with chronic hepatitis $\mathrm{C}$ and normal aminotransferase levels. Gastroenterology 127: 1724-1732, 2004.

21. Manigold T, Rehermann B. Chronic hepatitis B and hepatocarcinogenesis: does prevention of "collateral damage" bring the cure? Hepatology 37: 707-710, 2003.

22. Mochizuki K, Hayashi N, Hiramatsu N, et al. Fas antigen expression in liver tissues of patients with chronic hepatitis B. J Hepatol 24: 1-7, 1996.

23. Kerr JF, Cooksley WG, Searle J, et al. The nature of piecemeal necrosis in chronic active hepatitis. Lancet 2: 827-828, 1979.

24. Lawson JA, Fisher MA, Simmons CA, et al. Parenchymal cell apoptosis as a signal for sinusoidal sequestration and transendothelial migration of neutrophils in murine models of endotoxin and Fas-antibody-induced liver injury. Hepatology 28: 761-767, 1998.

25. Jacobson IM, Ahmed F, Russo MW, et al. Interferon alfa-2b [correction of alpha-2b] and ribavirin for patients with chronic hepatitis C and normal ALT. Am J Gastroenterol 99: 1700-1705, 2004.

26. Calabrese F, Pontisso P, Pettenazzo E, et al. Liver cell apoptosis in chronic hepatitis $\mathrm{C}$ correlates with histological but not biochemical activity or serum HCV-RNA levels. Hepatology 31: 1153$1159,2000$.

27. Jaeschke H, Lemasters JJ. Apoptosis versus oncotic necrosis in hepatic ischemia/reperfusion injury. Gastroenterology 125: 12461257, 2003.

28. Faouzi S, Burckhardt BE, Hanson JC, et al. Anti-Fas induces hepatic chemokines and promotes inflammation by an NF-kappa Bindependent, caspase-3-dependent pathway. J Biol Chem 276: 49077-49082, 2001.

29. Casini A, Ceni E, Salzano R, et al. Neutrophil-derived superoxide anion induces lipid peroxidation and stimulates collagen synthesis in human hepatic stellate cells: role of nitric oxide. Hepatology 25: 361-367, 1997.

30. Clippinger AJ, Gearhart TL, Bouchard MJ. Hepatitis B virus X protein modulates apoptosis in primary rat hepatocytes by regulating both NF-kappaB and the mitochondrial permeability transition pore. J Virol 83: 4718-4731, 2009.

31. Du J, Liang X, Liu Y, et al. Hepatitis B virus core protein inhibits TRAIL-induced apoptosis of hepatocytes by blocking DR5 expression. Cell Death Differ 16: 219-229, 2009.

32. Nagai H, Matsumaru K, Feng G, et al. Reduced glutathione depletion causes necrosis and sensitization to tumor necrosis factoralpha-induced apoptosis in cultured mouse hepatocytes. Hepatol- ogy 36: 55-64, 2002.

33. Nakajima T, Kamijo Y, Tanaka N, et al. Peroxisome proliferatoractivated receptor alpha protects against alcohol-induced liver damage. Hepatology 40: 972-980, 2004.

34. Okiyama W, Tanaka N, Nakajima T, et al. Polyenephosphatidylcholine prevents alcoholic liver disease in PPAR alpha-null mice through attenuation of increases in oxidative stress. J Hepatol 50: 1236-1246, 2009.

35. Kawase T, Kato S, Lieber CS. Lipid peroxidation and antioxidant defense systems in rat liver after chronic ethanol feeding. Hepatology 10: 815-821, 1989.

36. Stephens NG, Parsons A, Schofield PM, et al. Randomised controlled trial of vitamin $\mathrm{E}$ in patients with coronary disease: Cambridge Heart Antioxidant Study (CHAOS). Lancet 347: 781-786, 1996.

37. von Herbay A, Stahl W, Niederau C, et al. Diminished plasma levels of vitamin $\mathrm{E}$ in patients with severe viral hepatitis. Free Radic Res 25: 461-466, 1996.

38. Rocchi E, Casalgrandi G, Ronzoni A, et al. Antioxidant liposoluble vitamins and carotenoids in chronic hepatitis. Eur J Intern Med 12: 116-121, 2001.

39. Kazzaz JA, Xu J, Palaia TA, et al. Cellular oxygen toxicity. Oxidant injury without apoptosis. J Biol Chem 271: 15182-15186, 1996.

40. Blatt NB, Glick GD. Signaling pathways and effector mechanisms pre-programmed cell death. Bioorg Med Chem 9: 1371-1384, 2001.

41. Allen RG, Tresini M. Oxidative stress and gene regulation. Free Radic Biol Med 28: 463-499, 2000.

42. Gonzalez R, Collado JA, Nell S, et al. Cytoprotective properties of alpha-tocopherol are related to gene regulation in cultured Dgalactosamine-treated human hepatocytes. Free Radic Biol Med 43: 1439-1452, 2007.

43. Tanaka N, Nagaya $T$, Komatsu $M$, et al. Insulin resistance and hepatitis C virus: a case-control study of non-obese, non-alcoholic and non-steatotic hepatitis virus carriers with persistently normal serum aminotransferase. Liver Int 28: 1104-1111, 2008.

44. Oh MK, Winn J, Poordad F. Diagnosis and treatment of nonalcoholic fatty liver disease. Aliment Pharmacol Ther 28: 503-522, 2008.

45. De Minicis S, Brenner DA. Oxidative stress in alcoholic liver disease: role of NADPH oxidase complex. J Gastroenterol Hepatol 23 (Suppl 1): S98-S103, 2008.

\footnotetext{
(C) 2009 The Japanese Society of Internal Medicine http://www.naika.or.jp/imindex.html
} 